\title{
Phase I and II Upgrades of the ATLAS Muon Spectrometer with Integrated Small Diameter Drift Tube Chambers and Thin-Gap Resistive Plate Chambers
}

\author{
Verena Walbrecht* \\ on behalf of the ATLAS Muon Collaboration \\ Max Planck Institute for Physics, Munich (Germany) \\ E-mail: walbrech@mpp.mpg • de
}

\begin{abstract}
The ATLAS muon spectrometer comprises excellent muon trigger capabilities and high muon momentum resolution up to the $\mathrm{TeV}$ scale. Yet, in a small region between the barrel and endcap parts of the muon spectrometer, a non-negligible muon trigger rate from charged particles not emerging from the pp interaction point has been observed. To prevent such fake triggers, the end of the inner ring of the muon spectrometer barrel in the barrel end-cap transition region, which is currently not equipped with trigger chambers, will be instrumented with resistive plate chambers (RPCs) in the next long shutdown of the LHC in 2019 and 2020. As the space in this region is extremely limited the present muon drift-tube chambers will have to be removed and replaced by an integrated system of thin-gap RPCs and small diameter muon drift-tube (sMDT) chambers. Final prototypes of both chambers have been successfully produced recently fulfilling all very tight spatial requirements and showing the expected performance. Results of mechanical measurements of the sMDT chamber geometry with a coordinate measurement machine show a wire positioning accuracy of better than $10 \mu \mathrm{m}$. The tests of the thin-gap RPC in a high-energy muon beam at CERN show full chamber efficiency and an unprecedented time resolution better than $0.5 \mathrm{~ns}$. In the conference contribution the design, construction, and tests of the new sMDT and RPC chambers, as well as the progress of series production, will be presented.
\end{abstract}

Sixth Annual Conference on Large Hadron Collider Physics (LHCP2018)

4-9 June 2018

Bologna, Italy

${ }^{*}$ Speaker. 


\section{Motivation}

The design of the muon spectrometer of the ATLAS detector [1] is shown in in Figure 1. The barrel part is build up of three concentric layers. The inner layer is located at radii about 5 , the middle at 8 and the outer at 10 metres. To provide precision measurements for the muon momenta, each of the three layers consists of Monitored Drift Tube (MDT) chambers (shown in dark green). In addition in the middle and outer layer the MDT chambers are equipped with Resistive Plate Chambers (RPC) (shown in red) to provide the high- $p_{T}$ muon trigger system, which is built out of a coincidence of all three layers. In the endcap the MDT chambers are mounted perpendicular to the beam axis. Here a different trigger chamber technology, the Thin Gap Chambers (TGC), has been chosen. The muon system is embedded in a toroidal magnetic field provided by eight superconducting coils in the barrel region $(\eta<1.7)$ and two further magnet systems in the end-cap region.

The high- $p_{T}$ muon trigger acceptance is limited to $\approx 72 \%$, due to partially instrumented regions of the muon spectrometer, as shown in Figure 1. In order to provide space for services of the inner detector and the calorimeters a partially instrumented region is located at $\eta=0$. Further partially instrumented regions are at $\eta=0.4,0.75$ and 1.0 , because of the toroid and rib structures.

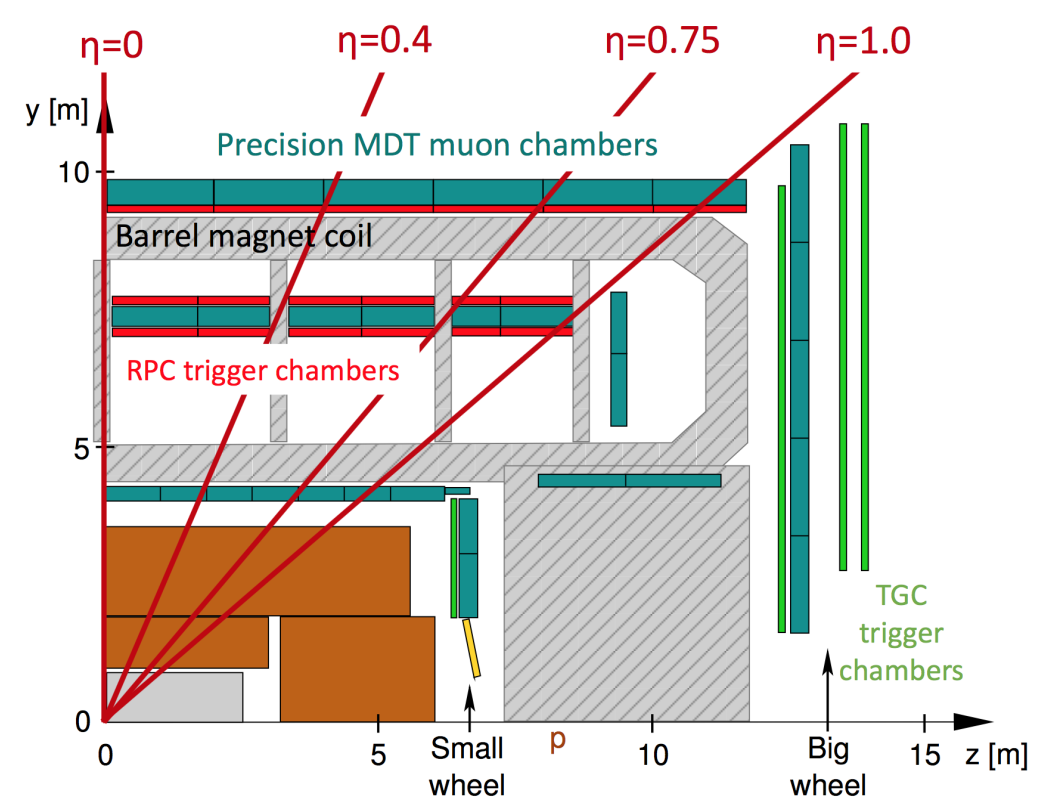

Figure 1: Cutaway trough the ATLAS Detector showing the partially instrumented regions of the muon spectrometer.

This limited muon trigger acceptance can be increased with the installation of additional Thin-Gap RPC chambers with increased high-rate capability in the inner barrel layer. Therefore the existing MDT chambers will be replaced with Small-Diameter Muon Drift Tube (sMDT) chambers in the small barrel sectors in order to free space for the RPCs [2]. 
In the beginning of 201712 sMDT chambers were installed (Phase I) in the middle layer of the feet region of the ATLAS muon spectrometer. In order to prevent non-negligible muon trigger rate from charged particles not emerging from the pp interaction point, which has been observed in a small region between the barrel and end-cap parts of the muon spectrometer, 16 new integrated muon tracking and trigger chambers (sMDT+RPC chambers) will be installed during the next long shutdown in 2019 and 2020 at the ends of the toroid magnet coils in the small sectors of the barrel inner layer (BIS) of the ATLAS muon spectrometer (Phase II).

\section{Small-Diameter Muon Drift Tube (sMDT)}

An sMDT has a reduced drift tube diameter of $15 \mathrm{~mm}$ compared to MDT's diameter of $30 \mathrm{~mm}$. This reduction by a factor of 2 leads to an eight times lower background occupancy, due to four times shorter maximum drift time and two times smaller tube cross section. The dead time of the electronics is reduced by a factor of 4 to the sMDT's maximum drift time.

The drift tube production is automated. First the wire is inserted by air flow as shown in Figure 2 (a). After that the endplug and the wire are fixed, shown in Figure 2 (b). The last step is the tensioning of the wire and the measurement of the wire tension, which is pictured in Figures 2 (c) and (d).

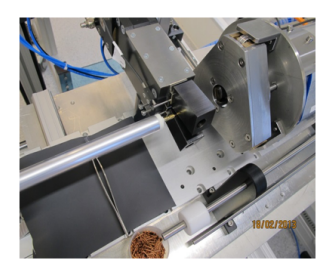

(a) Wire insertion by air flow.

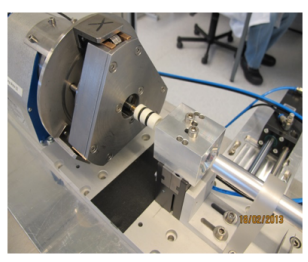

(b) Endplug and wire fixation.

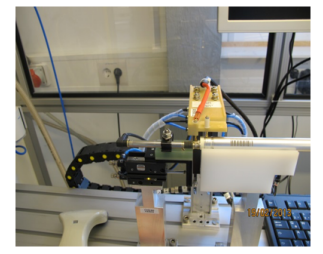

(c) Wire tensioning.

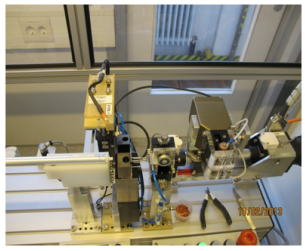

(d) Wire tension measurement.

Figure 2: Automated drift tube production at the Max-Planck-Institute for physics in Munich.

\section{Thin-Gap Resistive Plate Chambers}

The structure of the new Thin-Gap RPC Chambers used for the new integrated muon tracking and trigger chambers for the upgrade of the ATLAS muon spectrometer is shown in Figure 3. The twice thinner gas gaps $(1 \mathrm{~mm})$ and thinner high-pressure phenolic laminate (HPL) electrodes and new highly sensitive amplifiers improve the time resolution from $1 \mathrm{~ns}$ to $0.4 \mathrm{~ns}$. In addition the operation voltage can be substantially reduced from $9.6 \mathrm{kV}$ to $5.4 \mathrm{kV}$ and a 15 times lower gas gain and avalanche charge can be achieved. This leads to an increase in lifetime well beyond 10 years of operation at HL-LHC background rates. 


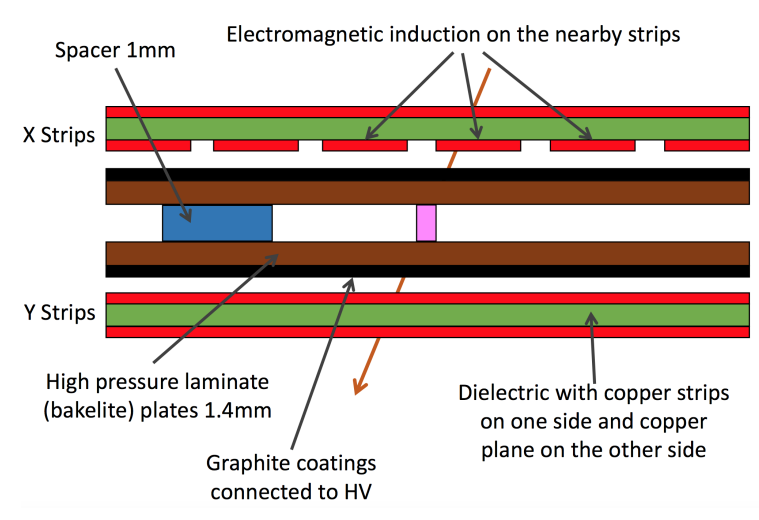

Figure 3: Structure of the Thin-Gap Resistive Plate Chambers used for the new integrated muon tracking and trigger chambers for the upgrade of the ATLAS muon spectrometer.

\section{Integrated sMDT and Thin-Gap RPC Chambers}

The integrated sMDT chambers have a height of $249 \mathrm{~mm}$ including the rail support and consists of $125 \mathrm{~mm}$ long drift tubes arranged in two multilayers, whereas one multilayer consists of four sMDT layers. In total the chambers are build up of either 648 or 744 drift tubes. Due to the overlap with the inner cap wheel, the chambers have complex shapes which vary from sector to sector. The trigger chambers have a triplet of gas gaps and are installed at the bottom of the chambers. Optical sensors are placed on the top and the bottom of sMDT drift tube layers for the global alignment system. An example of the design of the new integrated sMDT and Thin-Gap RPC Chambers is shown in Figure 4.

\section{Chamber Construction and Test}

Four of the 16 new integrated muon tracking and trigger chambers have already been assembled including the optical sensors for monitoring deformations and for the global alignment in the barrel. The required wire positioning accuracy of $20 \mu \mathrm{m}$ is verified by measuring the wire grid at both ends of the chamber with a feeler gauges on a flat granite table. To guarantee a precision measurement, the measurement is done on precisely machined reference surfaces at the endplugs of the drift tubes. For the assembled chambers an overall wire positioning accuracy of $15 \mu \mathrm{m}$ could be achieved (Figure 5 (a)).

In addition the time resolution of Thin-Gap RPC's has been tested (Figure 5 (b)) and an excellent time resolution better than $0.4 \mathrm{~ns}$ on a single hit has been measured.

\section{References}

[1] ATLAS Collaboration, 2008 JINST 3 S08003

[2] H. Kroha et. al., Nucl. Instrum. Meth. A845 (2017) 244-245 


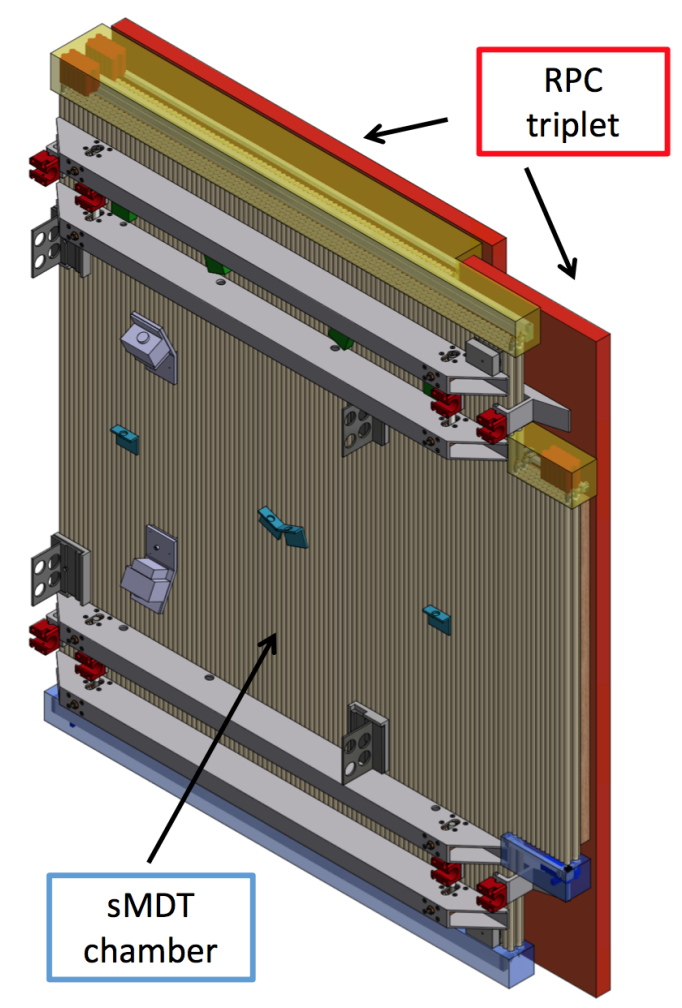

Figure 4: Design of the new integrated Small-Diameter Muon Drift Tube and Thin-Gap Resistive Plate Chambers.

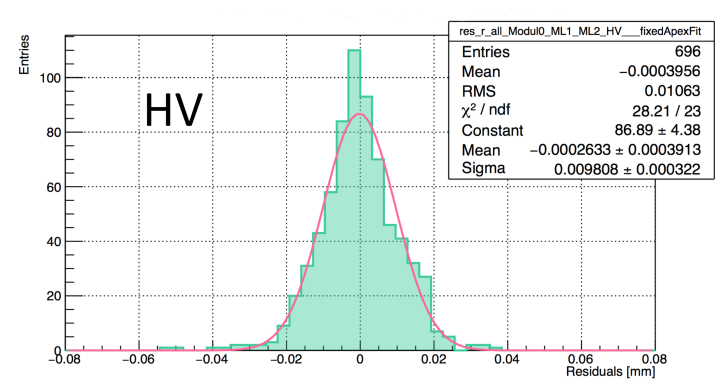

(a)

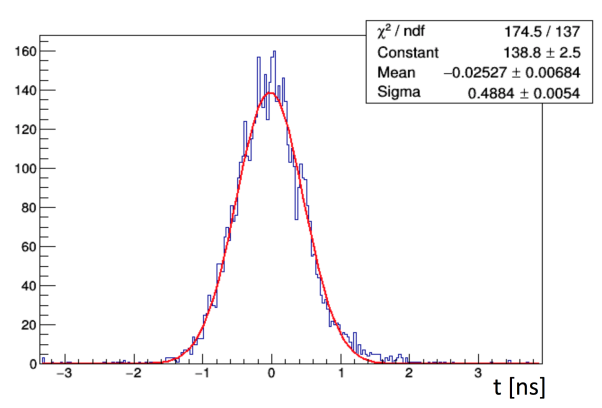

(b)

Figure 5: Measurement of the wire positioning accuracy transversal to tubular axle for the HV side (a) and of the time resolution (b). 